\title{
Influência Dietética e Nutricional na Migrânea
}

\section{Dietetics and Nutrition Influence in the Migraine}

\author{
Lívia Christine Santana e Silva*; Betânia de Jesus e Silva de Almendra Freitas ${ }^{\mathrm{b}}$
}

${ }^{a}$ Universidade Federal do Piauí, Curso de Nutrição.

bUniversidade Federal do Piauí, Centro de Ciências da Saúde.

*E-mail: liviachristine@hotmalil.com.

Recebido em: 10/06/2015; Aceito em: 16/11/2015

\begin{abstract}
Resumo
A migrânea é um tipo de cefaleia altamente incapacitante, mais prevalente no sexo feminino e apresenta vários gatilhos dietéticos possíveis. A literatura traz alimentos, bebidas e condimentos que podem desencadear ou agravar as crises de migrânea, por outro lado, alguns constituintes alimentares podem ser úteis no tratamento desta. Em relação ao estado nutricional, obesos e desnutridos apresentam crises de migrânea mais duradoras e frequentes que pessoas com peso adequado. O objetivo deste estudo foi relacionar fatores dietéticos e nutricionais com a migrânea. Para tanto, realizou-se uma revisão sistemática de literatura nas bases de dados Pubmed, Medline, SciELO e Google Acadêmico, além de livros técnicos sobre o tema. A identificação dos gatilhos alimentares revela-se uma das maneiras mais eficientes para a redução das crises de migrânea. Porém, tendo em vista que alguns alimentos saudáveis têm sido relatados como gatilhos desta comorbidade, desaconselha-se sua eliminação total da dieta. Além disso, a literatura também tem trazido diversos componentes nutricionais atuantes na prevenção da migrânea, tais como: lecitina, ácidos graxos ômega-3 e ômega-6, fenilalanina, taurina, metionina e isoflavona, algumas ervas (manjericão, melissa, gengibre, artemísia), riboflavina, coenzima Q10, magnésio, niacina, carnitina, ácido lipoico e cafeína. Reforça-se ainda a importância da manutenção de um estado nutricional adequado, pois a concentração de gordura abdominal e a desnutrição estão relacionadas a uma maior incapacidade decorrente da migrânea.
\end{abstract}

Palavras-chave: Cefaleia. Alimentação. Estado Nutricional.

\begin{abstract}
Migraine is a highly disabling type of headache, more prevalent in women and presents several possible dietary triggers. The literature provides food, beverages and seasonings that can trigger or aggravate migraine attacks, on the other hand, few food constituents may be useful in treatment of migraine. In relation to nutritional status, obese and malnourished have migraine attacks more long lasting and frequent than people with normal weight. The objective of this study was to relate dietary and nutritional factors with migraine in woman. Therefore, we carried out a systematic review of literature in the following databases Pubmed, Medline, SciELO and Google Scholar, and technical books on the topic. The identification of food triggers appears to be one of the most effective ways to reduce migraine attacks. However, given that some healthy foods have been reported as triggers of this comorbidity, we do not recommend their total elimination of the diet. In addition, the literature has also brought many nutritional components active in the prevention of migraine, such as lecithin, omega-3 and omega-6, fatty acids, phenylalanine, taurine, methionine and isoflavone, some herbs (basil, lemon balm, ginger, artemisia), riboflavin, co-enzyme Q10, magnesium, niacin, carnitine, caffeine, and lipoic acid. Is strengthened yet the importance of maintaining an adequate nutritional status, since the concentration of abdominal fat and malnutrition are related to greater disability resulting from the migraine.
\end{abstract}

Keywords: Headache. Feeding. Nutritional Status.

\section{Introdução}

A migrânea constitui-se em um tipo de cefaleia primária de alta prevalência, sendo precedida apenas pela cefaleia tipo tensional ${ }^{1}$. Além disso, ocupa no ranking da Organização Mundial de Saúde (OMS) a $19^{a}$ posição entre todas as doenças que causam incapacidade. E segundo a Sociedade Brasileira de Cefaleia $(\mathrm{SBCe})^{3}$, acomete em proporção mais mulheres do que homens. Caracteriza-se ainda como um tipo de cefaleia episódica, tipicamente unilateral e pulsátil, associada a queixas gastrointestinais e intolerância aos estímulos sensoriais.

A alimentação adequada exerce papel positivo no tratamento preventivo da migrânea, por outro lado, o jejum prolongado e o consumo de alguns alimentos, bebidas e condimentos têm sido relatados como os principais fatores desencadeantes desta. Além disso, o incentivo à alimentação adequada com vistas à perda de peso entre os obesos e ganho de peso entre os desnutridos deve ser uma preocupação no momento do atendimento ao paciente, uma vez que a prevalência de migrânea é maior nestes grupos ${ }^{4}$.

Tendo em vista a enorme importância que a alimentação tem sobre essa comorbidade, estudos enfocando a influência dietética e nutricional na migrânea revelam-se importantes ferramentas para ampliar o conhecimento de fatores envolvidos nesta condição patológica. Portanto, o presente estudo se justifica pela grande importância de se entender tal mecanismo, além de poder ser útil na adoção de proposições preventivas aptas a minimizar seu impacto nas condições de saúde e qualidade de vida de seus portadores, proporcionando 
o planejamento de novas pesquisas que visem melhorar a qualidade de vida desses pacientes.

Neste sentido, o presente estudo tem como objetivo relacionar fatores dietéticos e nutricionais com a migrânea.

\section{Desenvolvimento}

Realizou-se uma revisão de literatura nas bases de dados Pubmed, Medline, SciELO e Google Acadêmico, além de livros técnicos sobre o tema. Foram selecionados 286 artigos; após tradução dos títulos e leituras dos resumos dos mesmos, foram excluídos 234 artigos por abordarem o tema numa dimensão medicamentosa ou por outro tipo de tratamento e ainda por não permitir acesso na íntegra. Foram selecionados 48 artigos científicos relevantes publicados entre fevereiro de 1998 e dezembro de 2015; destes, 21 artigos foram publicados nos últimos quatro anos, disponíveis na íntegra, publicados nos idiomas português, inglês e espanhol, e que relatavam fatores dietéticos e nutricionais como gatilho da migrânea. Os descritores utilizados na pesquisa foram: migrânea, fatores dietéticos e estado nutricional. Para a elaboração dessa revisão, a leitura de resumos e títulos selecionados foi feita de forma independente por dois pesquisadores, assim como a leitura dos artigos incluídos e extração dos dados.

\subsection{Delimitação do tema}

A migrânea consiste em uma doença neurovascular, caracterizando-se por crises repetidas de cefaleia, com frequência bastante variável, podendo resultar em poucas crises durante toda a vida ou até diversos episódios a cada mês. O paciente acometido por essa comorbidade pode apresentar sintomas premonitórios, ou seja, antes da cefaleia, que consistem em: anorexia, dificuldade de concentração, irritabilidade, bocejos repetidos, alterações do humor, apetite ou sono, e distúrbios do sistema digestório ${ }^{5-7}$.

As crises de migrânea manifestam-se clinicamente por: cefaleia moderada a severa, de localização frontotemporal unilateral ou bilateral, em caráter pulsátil e/ou em pressão. Podendo ainda ser dividida em dois subtipos principais: migrânea sem ou com aura. Na migrânea sem aura, o paciente apresenta cefaleia recorrente, manifestando-se em crises que duram de 4 a 72 horas, a dor se apresentando localizada, unilateral, de caráter pulsátil, de intensidade moderada ou forte, exacerbada por atividade física rotineira e associada à fotofobia (intolerância à luz forte), fonofobia (intolerância a sons altos) e à osmofobia (intolerância a odores). A migrânea sem aura caracteriza-se ainda por ser o subtipo mais comum de migrânea, apresentando uma frequência de crises maior e geralmente mais incapacitante do que a migrânea com aura. Já esta última, é precedida de sintomas neurológicos focais, o paciente pode apresentar escotomas (área de cegueira parcial), alterações visuais, alterações motoras e/ou na fala ${ }^{5,8}$.

A prevalência anual de cefaleia crônica diária é de 1,7\% a 4\% da população adulta, em que a migrânea crônica representa aproximadamente metade dos casos. A migrânea, com ou sem aura, incluindo aguda e crônica, apresenta prevalência anual de $24,6 \%$ a $27,5 \%$ da população mundial ${ }^{9}$.

As crises de migrânea podem iniciar ainda na fase infantojuvenil, atingindo 3 a 10\% das crianças, afetando igualmente ambos os gêneros antes da puberdade, mas com predomínio em meninas após essa fase. Porém, cerca de 50\% das crianças podem ter remissão espontânea após a puberdade. Normalmente, apresenta um pico de prevalência aos 40 anos de idade, porém, menos frequente e aguda, após os 50 anos. E o aparecimento da migrânea após esta idade levanta a suspeita de cefaleia secundária como causa, acompanhando o paciente por toda a sua vida ${ }^{10-13}$.

A constatação de que a migrânea ocorre em membros de uma mesma família sugere que fatores genéticos estejam implicados no desenvolvimento das $\operatorname{crises}^{6,15}$. Aproximadamente, $50 \%$ das crianças que sofrem de migrânea têm, pelo menos, um dos pais acometidos por ela, podendo apresentar ainda pelo menos um familiar de primeiro grau acometido em $85 \%$ dos casos $^{6}$.

A migrânea apresenta fisiopatologia complexa e ainda insuficientemente conhecida. Estudos trazem a hipótese de que a migrânea é um distúrbio multifatorial, tendo a participação de mais de um nível do sistema nervoso, sendo as principais estruturas envolvidas: o sistema nervoso central (córtex e tronco cerebral), o sistema trigeminovascular (relação funcional das fibras trigeminais com os vasos), outras fibras autonômicas que inervam estes vasos, e os vários agentes vasoativos locais ${ }^{8,14,15}$.

Segundo Silva ${ }^{16}$, alguns fatores oriundos da prática clínica sugerem que as crises de migrânea estão diretamente relacionadas com alterações vasculares. Esta conclusão se baseia no fato de que, durante as crises, as artérias do couro cabeludo encontram-se dilatadas e hiperpulsáteis. Além disso, observou-se que a compressão digital da artéria carótida externa no pescoço ou da artéria temporal superficial do lado doloroso tem como mecanismo de ação a redução do fluxo de sangue para os vasos extracranianos e o uso de gelo sobre a região dolorida está diretamente relacionado com a vasoconstrição local.

Porém, Rodrigues ${ }^{6}$ sugere dois mecanismos como geradores dos fenômenos dolorosos na migrânea: fenômenos de sensibilização do sistema trigeminovascular e a inflamação neurogênica. Em concordância, Diener êt $a l \cdot{ }^{14}$ relatam que a hipersensibilidade central do complexo trigemenovascular aumenta a excitabilidade ou reduz os mecanismos inibidores da dor; relatam ainda que a estimulação trigeminal provoca liberação de neurotransmissores, que podem ser vistos como "marcadores" da atividade trigeminal, e sua detecção indica ativação deste sistema, são estes: o Peptídeo Relacionado ao Gene da Calcitonina (CGRP) e a Substância P (SP).

Dependendo do leito, a SP provoca vasodilatação mais modesta e relativamente mais curta, podendo atuar como 
neuromodulador do tono vascular. Enquanto o CGRP é um dos vasodilatadores mais potentes, podendo causar vasodilatação relativamente intensa e longa. Constatou-se ainda que essas substâncias possam ser liberadas nas meninges pela inflamação neurogênica. Além disso, a excitação do sistema trigeminal difunde este processo de inflamação, enquanto os estímulos trigeminais atingem o tálamo e posteriormente o córtex cerebral, contribuindo para a dor ${ }^{15}$. Ainda são necessários mais estudos para elucidarem completamente esse processo.

\subsection{Migrânea na mulher}

As razões para a preponderância de cefaleia na mulher ainda não são bem entendidas, mas suspeita-se de alguma relação com o hormônio feminino, vez que há tendência de aumento na incidência desta durante o período fértil e, redução após a menopausa ${ }^{5}$.

Aproximadamente $15 \%$ das mulheres tendem a experimentar sua primeira crise de migrânea por ocasião da menarca e, $50 \%$ das mulheres associam a migrânea aos ciclos menstruais, sendo esta a cefaleia mais importante para mulher, que se define por crises de migrânea sem aura, sem disfunção visual ou sem formigamentos nos braços e nas pernas, que ocorrem, exclusivamente, no período compreendido entre dois dias antes do início da menstruação até o final do fluxo menstrual e em nenhum outro período do ciclo menstrual ${ }^{5}$.

A contracepção segura e eficaz é essencial para todas as mulheres em idade fértil, mas anticoncepcionais orais combinados têm sido associados com o agravamento das crises e com o maior risco cardiovascular em mulheres com migrânea. Desta forma a literatura sugere a pílula contendo somente progestagênio (progesterona) como possível alternativa para a contracepção dessas pacientes ${ }^{17}$.

Durante a gravidez existe a possibilidade de alteração na intensidade da migrânea, podendo piorar, melhorar, desaparecer ou até mesmo iniciar-se. A sua melhora a partir do segundo trimestre pode ser de até $60 \%$, portanto é possível que a maioria das pacientes não necessite de tratamento das crises $^{5}$.

Para Bernstein e Mcardle ${ }^{18}$, a falta de períodos menstruais após a menopausa, pode induzir à melhora nos sintomas da migrânea. Cerca de 67,0\% das mulheres observaram, com o avançar da idade, amenização e redução na frequência de suas crises durante e após a menopausa. Porém, nos estudos de Dalton ${ }^{19}$, mulheres que se encontravam no período pósmenopausa e que realizaram histerectomia apresentaram crises de migrânea mensais semelhantes às mulheres que menstruavam regularmente, sinalizando que seu ciclo menstrual, regulado pelo hipotálamo no cérebro, ainda está funcionando. $\mathrm{A} \mathrm{SBCe}^{5}$ reforça que a prevalência aumentada de migrânea na mulher não pode ser exclusivamente relacionada ao período fértil, visto que as diferenças entre os sexos persistem na terceira idade.

Verifica-se ainda que procedimentos ginecológicos como histerectomia, dilatação, curetagem e cesariana aumentam a prevalência de migrânea nas mulheres, até mesmo nas que nunca tinham experimentado qualquer crise antes ${ }^{20}$.

\subsection{Migrânea e estado nutricional}

Tem-se evidenciado relação entre estado nutricional e migrânea, demonstrando que a prevalência desta enfermidade aumenta entre as pessoas desnutridas assim como entre as obesas $^{5}$; vários estudos mostram uma forte relação entre a obesidade e o excesso de peso e a migrânea ${ }^{21,22}$.

Peres et al. ${ }^{23}$ observaram que cefaleias primárias são mais comuns e incapacitantes em pacientes obesos, sendo a migrânea o diagnóstico mais frequente nesta população. Constatase ainda que a obesidade pode promover a cronificação da migrânea episódica ${ }^{24}$. Complementando esta ideia, Bigal e Lipton $^{25}$ encontraram uma razão de probabilidade de evolução para migrânea crônica de 1,4 em pacientes com sobrepeso, 1,7 em obesos e 2,2 em obesos mórbidos, comparados àqueles com peso normal.

Alguns estudos encontraram associação entre o IMC elevado e um maior risco para migrânea grave e cefaleia crônica diária ${ }^{26,27}$. Além disso, Bigal et al. ${ }^{28}$ encontraram maior prevalência e maior frequência (10-14 dias/mês) de migrânea em 7,4\% dos que apresentaram excesso de peso, em $8,2 \%$ dos obesos, e 10,4\% dos obesos mórbidos, em comparação com apenas $6,5 \%$ dos pacientes eutróficos. O grau de incapacidade gerado pela migrânea também variou em função do IMC, atingindo $32 \%$ naqueles com normalidade de peso, 37,2\% entre os que apresentaram excesso de peso, $38,4 \%$ dos obesos e 40,9\% dos indivíduos com obesidade mórbida.

Em um estudo realizado com mulheres, Winter et al. ${ }^{29}$ constataram que o IMC maior ou igual a $35 \mathrm{~kg} / \mathrm{m}^{2}$ aumentou o risco para migrânea com frequência diária em relação à migrânea ocasional. Nas mulheres com migrânea diária, o IMC maior ou igual a $35 \mathrm{~kg} / \mathrm{m}^{2}$ foi associado ao risco aumentado de fonofobia e fotofobia. Constatando-se que elevados valores de IMC condicionam não só a maior frequência de cefaleia migranosa, mas também as manifestações clínicas mais graves e desconfortáveis do quadro. Porém, apesar de Kinik et al..$^{30}$ observarem relação positiva entre o IMC e a frequência de cefaleias, a obesidade não teve influência sobre os sintomas associados à migrânea, incluindo: aura, fonofobia, fotofobia, náuseas e vômitos.

Reforçando a influência da obesidade sobre a migrânea, Verrotti et $a l .{ }^{31}$, observaram em seus estudos que a redução do peso, do IMC e da medida de circunferência da cintura provocaram diminuição da frequência e da intensidade das crises, da necessidade de uso de medicamentos agudos, e do grau de incapacidade, confirmando os benefícios da perda de peso no tratamento da migrânea.

A literatura tem comprovado que a associação entre migrânea e obesidade revela-se um fator de risco para outras comorbidades. Bond et al. ${ }^{32}$ observaram, em seus estudos, 
que um quarto dos participantes com migrânea e obesidade relataram catastrofização clínica, a qual se configura na antecipação pessimista do futuro ${ }^{33}$. Estes indivíduos tiveram crises mais frequentes, com maior cronicidade, duração mais longa, maior sensibilidade à dor, cefaleia de maior impacto e menor gestão de auto-eficácia ${ }^{32}$.

Além disso, os estudos de Janoska et al. ${ }^{34}$ verificaram correlação positiva entre colesterol total e triglicerídeo elevados e maior frequência das crises de migrânea em mulheres. Desta forma, tem-se que o perfil lipídico pode exercer influência sobre a severidade da migrânea e contribui para o aumento do risco de doenças cardiovasculares. Temse comprovado maior risco cardiometabólico nas mulheres grávidas portadoras de migrânea, estas apresentam elevadas probabilidades de desenvolver dislipidemias, particularmente hipercolesterolemia e tal associação foi mais pronunciada entre as pacientes obesas ${ }^{35}$.

\subsection{Fatores dietéticos desencadeantes das crises de migrânea}

A migrânea não é necessariamente caracterizada pela presença de cefaleia, mas pelo estado de susceptibilidade constante a esta, o que torna o migranoso permanentemente sujeito a uma crise, mediante fatores desencadeantes. Neste sentido, os fatores dietéticos encontram-se entre os mais comuns fatores desencadeantes, porém deve-se levar em conta que a resposta a estes diferem entre os pacientes ${ }^{6,15,36}$.

Os gatilhos alimentares podem afetar sua fisiopatologia, influenciando na liberação de serotonina e noradrenalina, causando vasoconstrição ou vasodilatação, ou por estimulação direta de gânglios trigeminal, tronco cerebral e vias neuronais corticais, contribuindo assim para o desencadeamento de migrâneas em pessoas susceptíveis. Porém, os mecanismos biológicos que explicam a ação e precisam desses gatilhos na precipitação da migrânea permanecem obscuros ${ }^{36,37}$.

Muitos estudos associam alimentos, bebidas e aditivos com a migrânea, sendo os mais citados: queijo, chocolate, frutas cítricas, banana, nozes, carnes "curadas", produtos lácteos, cereais, feijão, cachorro-quente, pizza, alimentos gordurosos, sorvetes, frutos do mar, ovos, chá, refrigerantes, café e bebidas alcoólicas, especialmente o vinho tinto e a cerveja e aditivos alimentares, como nitrato de sódio, glutamato monossódico e aspartame $\mathrm{e}^{37-39}$. Além disso, têm-se ainda como fatores desencadeantes alguns comportamentos alimentares, tais como: privação de cafeína, privação ou baixo consumo de líquidos e jejum prolongado. Como se pode ver, aparentemente, o alimento, em si, não é o responsável direto pelas crises de migrânea, mas sim, alguns componentes alimentares que, por sua vez, estão presentes em mais de um tipo de alimento ${ }^{39,40}$.

Dentre os gatilhos dietéticos mais citados e suas fontes alimentares, respectivamente, encontram-se: álcool (bebidas alcoólicas); aspartame e sucralose (adoçantes artificiais, chicletes, produtos diet e light); glutamato monossódico (comida chinesa, temperos prontos, alimentos industrializados, embutidos); cafeína (café, chá, chocolate, refrigerantes à base de cola); histamina (alimentos fermentados, como o chucrute e alimentos em conserva, como as sardinhas); nitratos e nitritos (carnes curadas e embutidas, como salsicha e mortadela); octamina e dopamina (frutas cítricas, banana, ameixa vermelha, figos, passas, abacate); aminas vasoativas (tiramina e feniletilamina); chocolate, queijo cheddar, queijo camembert, levedo de cerveja, peixe em conserva, vinho tinto, cerveja, vagens, café 7 .

Nos estudos de Rockett et al. ${ }^{39}$ foi detectada a presença de diversos fatores dietéticos como desencadeantes das crises de migrânea, e apenas $2,4 \%$ dos pacientes não se queixaram de qualquer fator dietético como desencadeante. Mais de 90\% dos pacientes relataram a susceptibilidade a no mínimo cinco desses fatores, sendo o jejum o gatilho mais frequente. Em concordância com esse estudo, Fukui et al. ${ }^{41}$ encontraram que $83,5 \%$ dos participantes apresentaram algum fator alimentar como desencadeante, sendo o jejum o mais frequente $(63,5 \%)$, seguido pela ingestão de álcool, chocolate e cafeína.

O jejum prolongado tem sido relatado como um dos principais fatores desencadeantes para a migrânea. Isso pode estar relacionado à hipoglicemia, pois o cérebro é incapaz de funcionar adequadamente nessa condição, uma vez que tem como principal fonte de energia a glicose. Como resultado, o fluxo sanguíneo para o cérebro aumenta para captar mais glicose e os tecidos nervosos se tornam mais sensíveis à dilatação dos vasos sanguíneos, podendo assim provocar uma crise de migrânea ${ }^{7}$.

Kokavec $^{42}$ observou em sua pesquisa que após o consumo de sacarose os níveis de insulina no soro foram significativamente superiores nas mulheres com migrânea; enquanto o nível de cortisol no soro foi significativamente superior em homens com migrânea. Observando assim que os efeitos da sacarose em biomarcadores do metabolismo e a utilização de energia não são os mesmos para homens e mulheres com migrânea, sugerindo que os fatores subjacentes da patogenia da migrânea também sejam diferentes dependendo do sexo de seu portador.

Um estudo realizado com 3.069 mulheres com e sem cefaleia ou migrânea grave sugeriu não haver diferenças nos padrões de consumo alimentar entre as mulheres com e sem migrânea, porém, quanto à qualidade alimentar, verificouse que mulheres eutróficas com migrânea apresentaram significativamente menor qualidade da dieta do que as mulheres sem migrânea $(\mathrm{p}<0,0001)^{43}$.

O tratamento nutricional, descrito pela literatura, consiste em dieta de exclusão, ou seja, recomenda-se identificar os fatores dietéticos relacionados à migrânea e eliminá-los da dieta. Porém, deve-se ressaltar que nem todos os portadores de migrânea são, obrigatoriamente, sensíveis a todos ou qualquer um destes alimentos, nem irão desencadear, precisamente, 
uma crise cada vez que consumi-los ${ }^{7}$.

\subsection{Fatores nutricionais atuantes na prevenção da migrânea}

Tendo em vista que o grande número de drogas e classes diferentes de substâncias empregadas no tratamento preventivo da migrânea não têm se mostrado inteiramente eficazes e que, além disto, muitas destas substâncias podem levar entre 40 e 50 dias para demonstrar alguma eficácia clínica, o uso de tratamentos não medicamentosos torna-se bem oportuno no sentido de amenizar as crises e potencializar o tratamento, além de ser eficaz na diminuição do uso de fármacos, eliminando assim os possíveis efeitos colaterais decorrentes deles ${ }^{8}$.

Para melhorar o metabolismo cerebral em geral, alguns nutrientes, como: lecitina, ácidos graxos ômega-3 (n-3), fenilalanina, taurina, metionina e isoflavona, algumas ervas (manjericão, melissa, gengibre, artemísia) e nutrientes que têm efeito positivo no metabolismo mitocondrial demonstraram ser eficazes no tratamento da migrânea, dentre estes incluemse: a riboflavina (vitamina B2), coenzima Q10 (CoEQ10) (também conhecida como ubiquinona), magnésio, niacina, carnitina, e ácido lipoico. Porém, mais estudos são necessários para entender a relação do metabolismo mitocondrial na fisiopatologia da migrânea ${ }^{7,44}$.

Apesar de alguns estudos trazerem a cafeína como desencadeante das crises de migrânea ${ }^{7,39,41}$, o seu uso para o tratamento da cefaleia migranosa sem aura parece estar relacionado à eficácia analgésica, além de aumentar e prolongar o efeito analgésico de outros fármacos prescritos para essa patologia, por meio de interações farmacodinâmicas ${ }^{45}$.

Schoenen et $a l .{ }^{46}$ observaram que a suplementação diária de $400 \mathrm{mg}$ de riboflavina proporcionou melhora na migrânea em mais de $50 \%$ dos portadores, sem efeitos colaterais. A riboflavina é encontrada em pequenas quantidades em muitos alimentos e pode aumentar a produção de energia nos vasos sanguíneos cerebrais, e assim como a coenzima Q10, apresenta função antioxidante. Os estudos de Rozen et al. ${ }^{47}$ também constataram a eficácia da coenzima Q10 no tratamento preventivo da migrânea, pois $61,3 \%$ dos pacientes tiveram redução superior a $50 \%$ na frequência de crises.

O magnésio é um mineral importante que está envolvido em várias funções celulares. A sua deficiência apresenta papel importante na patogênese da migrânea, promovendo depressão cerebral alastrante, alteração da liberação de neurotransmissores e hiperagregação plaquetária. Dado este papel multifacetado do magnésio na migrânea, o seu uso, tanto no tratamento agudo quanto preventivo, tem sido pesquisado como uma opção potencialmente simples, barata, segura e bem tolerada ${ }^{48}$.

Esses achados corroboram com o estudo de Gaul et. $a l^{49}$, que objetivou avaliar a eficácia de um suplemento nutricional contendo uma combinação fixa de magnésio, riboflavina e coenzima Q10 como tratamento profilático para migrânea. Os resultados indicaram que a intensidade da cefaleia migranosa foi significativamente reduzida no grupo suplementado em comparação com o placebo $(p=0,03)$. E a avaliação da eficácia do tratamento por paciente também foi melhor no grupo suplementado em comparação com o placebo $(p=0,01)$, observando assim que o tratamento teve impacto positivo na frequência de migrânea.

$\mathrm{O}$ ácido graxo ômega 3 tem efeitos benéficos sobre as reações inflamatórias e a produção de citocinas, podendo ser utilizado para combater a inflamação neurogênica e diminuir a liberação de serotonina pelas plaquetas, além de apresentar atividade vasorrelaxante, agindo assim no tratamento profilático da migrânea. Tem-se ainda que ensaios controlados com placebo, usando óleo de peixe em doenças inflamatórias crônicas, revelaram benefícios significativos, incluindo a diminuição da atividade dessa patologia, além de requererem menor utilização de drogas anti-inflamatórias ${ }^{50,51}$.

Ramsden et al. ${ }^{52}$ observaram que a suplementação conjunta de ácidos graxos ômega 3 e ômega 6 mostrou-se eficaz para induzir reduções na duração e frequência das cefaleias, quando comparado ao grupo suplementado apenas com ômega 6. Observou-se ainda que a intervenção dietética com esses ácidos graxos reduziu a intensidade das dores de cabeça, alterou mediadores lipídicos antinociceptivos, além de melhorar a qualidade de vida dos pacientes. Além disso, Tajmirriahi et al..$^{53}$ constataram redução significativa na duração, frequência mensal e gravidade das crises de migrânea com a suplementação dietética com óleo de peixe. Estes dados corroboram o estudo de Harel et al. ${ }^{54}$, que alcançou os mesmos resultados com óleo de peixe e azeite de oliva. Sugerindo-se que esses óleos são benéficos no tratamento da migrânea.

\section{Conclusão}

A migrânea revela-se uma doença complexa e de fisiopatologia ainda insuficientemente conhecida; a preponderância na mulher está possivelmente relacionada aos hormônios femininos. A relação entre estado nutricional e migrânea revela que tanto a desnutrição quanto o sobrepeso e a obesidade são fatores agravantes desta comorbidade. Desta forma, um programa de ganho ponderal para os desnutridos, ou que estejam com baixo peso, e perda de peso para obesos, ou que estejam com sobrepeso, revela-se uma estratégia importante no tratamento e amenização das crises de migrânea.

Verificam-se muitos alimentos envolvidos no desencadeamento e/ou agravamento das cefaleias migranosas. Nesse sentido, a identificação dos gatilhos alimentares revelase uma das maneiras mais eficientes para a redução das crises de migrânea. Uma dieta de eliminação simultânea de todos os potenciais gatilhos alimentares não é aconselhável na prática, pois alguns dos alimentos citados como gatilhos, apresentam nutrientes indispensáveis para uma alimentação saudável. Além disso, comportamentos alimentares como 
jejum prolongado e ausência de refeições também estão envolvidos com as crises de migrânea, ressaltando, desta forma a importância da reeducação alimentar feita por um profissional de nutrição.

O tratamento não medicamentoso tem apresentado resultados positivos na literatura, podendo servir como base para a criação de protocolos de suplementação de vitaminas, minerais e componentes dietéticos com poucos efeitos colaterais, quando comparados com as drogas medicamentosas. No entanto, análises prospectivas são necessárias para estabelecer a participação específica dos gatilhos dietéticos e o grau de interferência do estado nutricional sobre os sintomas da migrânea.

\section{Referências}

1. Nitrini R, Bacheschi LA. A neurologia que todo médico deve saber. São Paulo: Atheneu; 2003.

2. OMS - Organização Mundial de Saúde. Relatório Mundial de Saúde. Saúde mental: nova concepção, nova esperança. Genebra: WHO; 2001.

3. SociedadeBrasileiradeCefaleia.Tiposdedordecabeça.[acesso em 8 dez 2014]. Disponível em http://www.sbce.med.br/ index.php?option $=$ com_content $\&$ view $=$ article $\& i d=23$ :tiposde-dor-de-cabeca\&catid $=19 \&$ Itemid=718\&showall=1\&limit start.

4. Felipe MR, Campos A, Vechi G, Martins L. Implicações da alimentação e nutrição e do uso de fitoterápicos na profilaxia e tratamento sintomático da enxaqueca: uma revisão. Nutr Rev Soc Bras Aliment Nutr 2010;35(2):165-79.

5. Sociedade Brasileira de Cefaleia - Comitê ad hoc. Recomendações para o tratamento profilático da migrânea: consenso da sociedade brasileira de cefaleia. Arq Neuropsiquiatr 2002;60(1):159-69.

6. Rodrigues MFM. Cefaleias na infância e adolescência: a enxaqueca migranosa e a cefaleia do tipo-tensão. Covilhã: Universidade da Beira Interior; 2008.

7. Iglesias HCE, Bottura R, Naves MMV. Fatores nutricionais relacionados à enxaqueca. Com Ciências Saúde 2009;20(3):229-40.

8. Krymchantowski AV, Moreira Filho PF. Atualização no tratamento profilático das enxaquecas. Arq Neuropsiquiatr 1999;57(2B).

9. Stovner LJ, Hagen K, Jensen R, Katsarava Z, Lipton R, Scher $\mathrm{A}$, et al. The global burden of headache: a documentation of headache prevalence and disability worldwide. Cephalalgia 2007;27:193-210.

10. Mueller LL. Diagnosing and managing migraine headache. J Am Osteopath Assoc 2007;107(10):10-6.

11. Wannmacher L, Ferreira MBC. Enxaqueca: mal antigo com roupagem nova. Uso racional de medicamentos: temas selecionados. Brasília: OPAS; 2004.

12. Peterlin BL, Scher AI. Migraine and the social selection vs causation hypotheses: a question larger than either/or? Neurology 2013;81(11):942-3.

13. Freitag FG. Why do migraines often decrease as we age? Curr Pain Headache Rep 2013;17(10):366-71.

14. Diener HC, Dodick DW, Goadsby PJ, Bigal ME, Bussone G,
Silberstein SD, et al. Utility of topiramate for the treatment of patients with chronic migraine in the presence or absence of acute medication overuse. Cephalalgia 2009;29(10):1021-7.

15. Vincent MB. Fisiopatologia da enxaqueca. Arq Neuropsiquiatr 1998;56(4):841-51.

16. Silva WF. Diagnóstico das cefaleias. São Paulo: Lemos; 2003.

17. Allais G, Chiarle G, Bergandi F, Benedetto C. The use of progestogen-only pill in migraine patients. Expert Rev Neur 2015. doi:10,1586/14737175.2016.1127161.

18. Bernstein C, Mcardle E. The migraine brain. New York: Souvenir; 2010.

19. Dalton, K. Hormonal aspects of migraine in women. Migraine Action Newsletter. 2001. Disponível em http://www.webmd. com/migraines-headaches/guide/hormones-headaches.

20. Arumugam M, Parthasarathy V. Increased incidence of migraine in women correlates with obstetrics and gynaecological surgical procedures. Int J Surg 2015;22(1): 1059.

21. Bond DS, Roth J, Nash JM, Wing RR. Migraine and obesity: epidemiology, possible mechanisms and the potential role of weight loss treatment. Obes Rev 2011;12(5):362-71.

22. Peterlin BL, Rosso AL, Williams MA, Rosenberg JR, Haythornthwaite JA, Merikangas KR, et al. Episodic migraine and obesity and the influence of age, race, and sex. Neurology 2013;81(15):1314-21.

23. Peres MFP, Lerário DDG, Garrido AB, Zukerman E. Cefaleias primárias em pacientes obesos. Arq Neuropsiquiatr 2005;63(4):931-33.

24. Young WB. Preventive treatment of migraine: effect on weight. Curr Pain Headache Rep 2008;12(3):201-6.

25. Bigal ME, Lipton RB. Obesity is a risk factor for transformed migraine but not chronic tension-type headache. Neurology 2006;67(2):252-7.

26. Chorążka K, Janoska M, Swić P, Domitrz I. Body mass index and serum lipid levels in effect on the incidence and course of migraine. Neurol Neurochir Pol 2013;47(6):572-6.

27. Scher AI, Stewart WF, Ricci JA, Lipton RB. Factors associated with the onset and remission of chronic daily headache in a population-based study. Pain 2003;106(1/2):81-9.

28. Bigal ME, Tsang A, Loder E, Serrano D, Reed ML, Lipton RB. Body mass index and episodic headaches: a populationbased study. Arch Intern Med 2007;167(18):1964-70.

29. Winter AC, Berger K, Buring JE, Kurth T. Body mass index, migraine, migraine frequency and migraine features in women. Cephalalgia 2009;29(2):269-78.

30. Kinik ST, Alehan F, Erol I, Kanra AR. Obesity and paediatric migraine. Cephalalgia 2010;30(1):105-9.

31. Verrotti A, Agostinelli S, D’Egidio C, Di Fonzo A, Carotenuto M, Parisi P, et al. Impact of a weight loss program on migraine in obese adolescents. Eur J Neurol 2013;20(2):394-7.

32. Bond DS, Buse DC, Lipton RB, Thomas JG, Rathier L, Roth J et al. Clinical Pain Catastrophizing in Women With Migraine and Obesity. Headache 2015;55(7):923-33.

33. Oliveira MIS. Intervenção cognitivo-comportamental em transtorno de ansiedade: relato de caso. Rev bras ter cogn 2011;7(1):30-3. 
34. Janoska M, Chorążka K, Domitrz I. Migraine frequency and its association with dyslipidemia in women. Neurol Neurochir Pol 2015;49(2):95-8.

35. Gelaye B, Larrabure-Torrealva GT, Qiu C, Luque-Fernandez MA, Peterlin BL, Sanchez SE et al. Fasting lipid and lipoproteins concentrations in pregnant women with a history of migraine. Headache 2015;55(5):646-57.

36. Millichap JG, Yee MM. The diet factor in pediatric and adolescent migraine. Pediatr Neurol 2003;28(1):9-15.

37. Saracco MG, Calabrese G, Cavallini M, Montano V, Rinaldi $\mathrm{B}$, Valfrè W, et al. Relationship between primary headache and nutrition: a questionnaire about dietary habits of patients with headache. Neurol Sci 2014;35(1):159-61.

38. Finocchi C, Sivori G. Food as trigger and aggravating factor of migraine. Neurol Sci 2012;33(1):77-80.

39. Rockett FC, Castro K, Oliveira VR, Perla AS, Chaves MLF, Perry IDS. Perceived migraine triggers: do dietary factors play a role? Nutr Hosp 2012;27(2):483-9.

40. Nazari F, Eghbali M. Migraine and its relationship with dietary habits in women. Iran J Nurs Midwifery Res 2012;17(2):6571.

41. Fukui PT, Gonçalves TR, Strabelli CG, Lucchino NM, Matos FC, Santos JP et al. Trigger factors in migraine patients. Arq Neuropsiquiatr 2008;66(3):494-9.

42. Kokavec A. Effect of sucrose consumption on serum insulin, serum cortisol and insulin sensitivity in migraine: evidence of sex differences. Physiol Behav 2015;142(1):170-8.

43. Evans EW, Lipton RB, Peterlin BL, Raynor HA, Thomas JG, O'Leary KC et al. Dietary intake patterns and diet quality in a nationally representative sample of women with and without severe headache or migraine. Headache 2015;55(4):550-61.

44. Yorns Junior WR, Hardison HH. Mitochondrial dysfunction in migraine. Semin Pediatr Neurol 2013;20(3):188-93.

45. Tavares C, Sakata RK. Cafeína para o tratamento de dor. Rev Bras Anestesiol 2012;62(3):394-401.
46. Schoenen J, Jacquy J, Lannerets M. Effectiveness of highdose riboflavin in migraine prophylaxis. A randomized controlled trial. Neurology 1998;50(2):466-70.

47. Rozen TD, Oshinsky ML, Gebeline CA, Bradley KC, Young WB, Shechter AL, et al. Open label trial of coenzyme Q10 as a migraine preventive. Cephalalgia 2002;22(2):137-41.

48. Mauskop A, Altura BM. Role of magnesium in the pathogenesis and treatment of migraines. Clin Neurosc 1998;5(1):24-7.

49. Gaul C, Diener HC, Danesch U; Migravent ${ }^{\circledR}$ Study Group. Improvement of migraine symptoms with a proprietary supplement containing riboflavin, magnesium and Q10: a randomized, placebo-controlled, double-blind, multicenter trial. J Headache Pain 2015;16(1):516.

50. Pradalier A, Bakouche P, Baudesson G, Delage A, CornailleLafage G, Launay JM, et al. Failure of omega-3 polyunsaturated fatty acids in prevention of migraine: a double-blind study versus placebo. Cephalalgia 2001;21(8):818-22.

51. Simopoulos AP. Omega-3 fatty acids in inflammation and autoimmune diseases. J Am Coll Nutr 2002;21(6):495-505.

52. Ramsden CE, Faurot KR, Zamora D, Suchindran CM, Macintosh BA, Gaylord S, et al. Targeted alteration of dietary n-3 and n-6 fatty acids for the treatment of chronic headaches: a randomized trial. Pain 2013;154(11):2441-51.

53. Tajmirriahi M, Sohelipour M, Basiri K, Shaygannejad V, Ghorbani A, Saadatnia W. The effects of sodium valproate with fish oil supplementation or alone in migraine prevention: a randomized single-blind clinical trial. Iran J Neurol 2012;11(1):21-4.

54. Harel Z, Gascon G, Riggs S, Vaz R, Brow W, Exil G. Supplementation with omega-3 polyunsaturated fatty acids in the management of recurrent migraines in adolescents. $J$ Adolesc Health 2002;31(2):154-61. 